\title{
Entre indicaciones geográficas y patrimonio inmaterial en el caso havana club
}

\author{
Between geographical indications and intangible \\ heritage in the havana club case
}

(iD) Janny Carrasco ${ }^{1}$

(9) Zadys Carrasco ${ }^{2}$

Resumen: La protección jurídica de las indicaciones geográficas (IGs) es un elemento indispensable en el equilibrio de la dicotomía local versus global. El objetivo es analizar la concepción internacional de las IGs y su relación con el patrimonio cultural inmaterial, exponiendo desafíos y oportunidades en el contexto de la legislación cubana y tomando como referencia el caso Havana Club. Para ello, definimos como la hipótesis del estudio que: Ias IGs en la realidad cubana constituyen un ejemplo de la necesidad de reinterpretar las IGs desde el papel del patrimonio cultural inmaterial y el papel del terruño en la materialización de su protección. La metodología empleada es de enfoque transdisciplinar y para ello se apoya en los métodos lógicos, histórico-sociales, culturales y jurídicos, suponiendo la combinación de métodos de las ciencias sociales y jurídicas en particular. Como resultado se concluye que la ampliación e inclusión del patrimonio cultural inmaterial y su interrelación con las

\footnotetext{
1 Professora de Direito Internacional e Direitos Humanos na FASF-LUZ, Minas Gerais. Pós-doutoranda na Universidade de Brasília 2019-2020. Doutora em Direito Internacional pela Universidade de Brasília 2018. Professora voluntaria de Direito Internacional Público pela Universidade de Brasília (2018-2019).Possui mestrado em Educação Superior - Comunicação Social pela Universidade Central Marta Abreu Las Villas (2010). Professora Assistente pela Universidade Central Marta Abreu Las Villas de 2007-2014.

2 Possui graduação em Engenharia Informatica - Universidad de las Ciencias Informáticas (2010). Mestre em Bioetica pelo Centro Diocesano de Formação Villa Clara- Universidade Catolica de Valencia San Vicente Martir(2014). Actuou como Analista de Estadistica na OficinaNacional de Estadistica de cuba (2010-2012). Analista de Sistema de Segurança Informátia da Asociação Hermanos Saíz Cuba(2012-2018)
} 
IGs, es esencial en la materialización de la protección jurídica de las IGs en el contexto cubano, pues permitirá proteger valores culturales locales e incrementar sus garantías jurídicas. El aporte del estudio radica en una nueva interpretación de las IGs delante del patrimonio cultural inmaterial.

Palabras claves: Indicaciones geográficas. Patrimonio cultural inmaterial. Terror. Havana club. Propiedad intelectual.

Resumo: A proteção legal das indicações geográficas (IGs) é um elemento indispensável para equilibrar a dicotomia local versus global. O objetivo é analisar a concepção internacional de IGs e sua relação com o patrimônio cultural imaterial, expondo desafios e oportunidades no contexto da legislação cubana e tomando como referência o caso Havana Club. Para tanto, definimos como hipótese do estudo que: as IGs na realidade cubana constituem um exemplo da necessidade de reinterpretar as IGs a partir do papel do patrimônio cultural imaterial e do papel da terra na materialização de sua proteção. A metodologia utilizada é de abordagem transdisciplinar e para isso conta com métodos lógicos, histórico-sociais, culturais e jurídicos, pressupondo a combinação de métodos das ciências sociais e jurídicas em particular. Como resultado, conclui-se que a expansão e inclusão do patrimônio cultural imaterial e sua inter-relação com as IGs é essencial na materialização da proteção jurídica das IGs no contexto cubano, pois permitirá a proteção dos valores culturais locais e aumentar suas garantias legais. A contribuição do estudo reside em uma nova interpretação das IGs sobre o patrimônio cultural imaterial.

Palavras chaves: Indicações geográficas. Patrimônio cultural imaterial. Terror. Havana club. Propriedade intelectual.

Abstract: The legal protection of geographical indications (GIs) is an
indispensable element in balancing the local versus global dichotomy.
The objective is to analyze the international conception of Gls and their
relationship with intangible cultural heritage, exposing challenges and
opportunities in the context of Cuban legislation and taking the Havana
Club case as a reference. For this, we define as the hypothesis of the 
study that: Gls in the Cuban reality constitute an example of the need to reinterpret Gls from the role of intangible cultural heritage and the role of the land in the materialization of its protection. The methodology used is of a transdisciplinary approach and for this it relies on logical, historical-social, cultural and legal methods, assuming the combination of methods from the social and legal sciences in particular. As a result, it is concluded that the expansion and inclusion of intangible cultural heritage and its interrelation with Gls is essential in the materialization of the legal protection of Gls in the Cuban context, as it will allow the protection of local cultural values and increase their legal guarantees. The study's contribution lies in a new interpretation of Gls in relation to intangible cultural heritage.

Keys words: Geographical indications. Intangible cultural heritage. Terror. Havana club. Intellectual property.

Data de submissão do artigo: Abril de 2021

Data de aceite do artigo: Novembro de 2021 
Entre indicaciones geográficas y patrimonio inmaterial en el caso havana club Janny Carrasco $\cdot$ Zadys Carrasco

\section{Introducción}

De acuerdo con la Organización Mundial de la Propiedad Intelectual (OMPI) la Indicación Geográfica es un signo utilizado en productos que tienen un origen geográfico concreto y poseen cualidades o una reputación derivada específicamente de su lugar de origen (OMPI, 2017, p. 4).

Digamos que las Indicaciones Geográficas (IGs) son protegidas por el sistema internacional de la propiedad intelectual para limitar las falsificaciones de productos que son claramente reconocidos por tener un vínculo territorial que determina su calidad y notoriedad, teniendo un impacto significativo en el precio del producto en el mercado internacional, así como un reconocimiento global por las características específicas que presentan en su conexión con el territorio de origen.

Tal como sucede con otras áreas de la propiedad intelectual, las IGs necesitan ser pensadas y construidas desde la perspectiva del patrimonio cultural inmaterial del territorio con el que guardan estrecha relaciona. Un análisis desde esta interdisciplinariedad pretende minimizar los impactos del escenario competitivo y globalizado que enfrentan los pequeños productores en el mercado internacional.

La protección jurídica de las IGs, desde la Propiedad Intelectual, es un fenómeno inevitable desde la lógica de la globalización, lo que resalta su significado económico y jurídico en la capacidad de transbordar las fronteras físicas de los Estados. Desde esta realidad es posible articular una crítica jurídica a dicha aplicación económica del derecho. En ese orden de cosas, el presente texto plantea la siguiente pregunta de investigación: ¿Es la protección jurídica de las IGs desde la realidad del patrimonio cultural inmaterial, la conexión esencial con el territorio de origen? La respuesta es que la integración del patrimonio cultural inmaterial y las IGs son cuestiones jurídicas indisolubles para sobrevivir en el 
Entre indicaciones geográficas y patrimonio inmaterial en el caso havana club Janny Carrasco $\cdot$ Zadys Carrasco

contexto global y que resaltan sin dudas el impacto de global de tradiciones locales y terruño.

Dicha respuesta, se fundamenta a modo de critica jurídica en tres pilares esenciales. El primero es el análisis de las IGs desde la perspectiva del derecho internacional y como trapazan las fronteras físicas e impactan a escala global. El segundo fundamento descansa en la lógica del patrimonio cultural inmaterial y su perspectiva internacional. El tercero demuestra la necesidad de entender las IGs desde el patrimonio cultural inmaterial, por el impacto que el "terror" o el terruño tiene en la producción de las IGs en el mercado internacional, tomando como referencia la legislación cubana y el caso Havana Club.

El contexto de este artículo busca pensar en la efectividad del sistema internacional de IGs y su relación con el patrimonio cultural inmaterial en el contexto cubano, así como el impacto directo del terruño en esta relación. Pensar en la protección jurídica de las IGs es algo que nos lleva a especular en las fragilidades del sistema internacionalysuimplementación en diferentes contextosjurídicos. Para mayor profundización de la protección jurídica de las IGs y a favor de la lógica internacional la doctrina internacional se muestra favorable a una protección jurídica divorciada desde la realidad del patrimonio cultural inmaterial, para lo cual las sociedades menos favorecidas en el comercio global se deparan con desafíos poco resueltos en su ámbito interno como es el caso de Cuba. En este orden de cosas, el presente texto contribuye a entender las IGs desde la realidad del patrimonio cultural inmaterial debido al valor del terruño en su protección e identificación internacional.

Metodológicamente, este texto abunda evidencias em dos líneas fundamentales, a saber, el caso Havana Club, para explicar la fragilidad del sistema internacional frente a los sistemas nacionales y la necesidad de su interconexión con el patrimonio cultural inmaterial, todo ello desde la realidad jurídica cubana. Si bien, esta evidencia no es, en modo alguno, conclusiva, es suficiente para apuntar las principales críticas al sistema internacional de las IGs y el patrimonio cultural inmaterial. 
Entre indicaciones geográficas y patrimonio inmaterial en el caso havana club Janny Carrasco • Zadys Carrasco

El escenario económico actual por el que circulan los productos cubanos denota la existencia de prácticas monopólicas que urgen de la necesidad de una protección más efectiva en relación a las IGs de productos que representan la cultura cubana en el ámbito internacional.

La necesidad de conceder protección a las IGs como elementos del patrimonio cultural inmaterial se apoya en tres afirmaciones. La primera es la conexión territorial deviene necesariamente el elemento representativo de cultura popular, maneras de hacer e idiosincrasia de un pueblo que vuelve suyo aquella creación por el valor agregado quele ofrece. La segunda es que la lógica del mercado ahoga la posibilidad de entender el enfoque multidisciplinar de las IGs, debido a las interpretaciones negligentes de la jurisprudencia de propiedad intelectual que descarta y hace evidente el divorcio preexistente entre estas y el patrimonio cultural inmaterial en el plano internacional. La tercera es la posibilidad de entender está interconexión como mecanismo de solución de conflictos en el caso "Havana Club". Finalmente, concluimos que el entendimiento interdisciplinar de las IGs y patrimonio cultural inmaterial es un proceso inevitable, por lo que la interpretación jurisprudencial debe ir caminando hacia ese sentido hasta no llegar las reformas legislativas internacionales y nacionales.

\section{Indicaciones Geográficas en la lógica del Derecho Internacional}

Entre las principales críticas que reciben las IGs destacamos la concepción de un derecho represor de la competencia desleal frente a conductas de engaño, falsedady uso indebido de reputación ajena. Por otro lado, un sistema de protección propia donde la exclusividad del derecho garantiza el monopolio de utilización y su vinculación con el patrimonio inmaterial es evidente en la mayoría de los casos. 
Entre indicaciones geográficas y patrimonio inmaterial en el caso havana club Janny Carrasco $\cdot$ Zadys Carrasco

Coincidiendo con (SANTIAGO, 2017, p. 26) "El término IGs se deriva de conceptos que han variado en diferentes instrumentos internacionales, por lo cual no se puede partir de una uniformidad en la terminología ni en los elementos constitutivos de las definiciones. La protección concreta varía tanto en función de la definición adoptada como de los intereses que se busca promover con su tutela.

Desde los Convenios de la Unión de Paris (CUP) 3 de $1883^{4}$, el Arreglo de Madrid de 18915; el Tratado de Lisboa de $1958^{6}$ y, por último, el Acuerdo de Propiedad Intelectual dedicado al Comercio (APDIC)de 1994 de la OMC, define expresamente en su artículo 22.1 "las indicaciones geográficas como aquellas que identifican un producto como originario del territorio de un Estado miembro de la OMC.

Una de las principales críticas a estos instrumentos internacionales recae en la denominación indistinta de Indicaciones Geográficas o Denominaciones de Origen, lo que dificulta para los operadores del derecho a nuestro criterio la uniformidad de definiciones ya que en ocasiones son utilizadas como sinónimos. Aunque por otro lado también favorece la protección de los productos originarios vinculados a un determinado territorio y que alcanza un impacto global.

En este sentido Blakeney resalta que: tanto en el Arreglo de Lisboa como en los Acuerdos de Propiedad Intelectual relacionados al Comercio la eficacia jurídica radica en proteger el origen de un producto que resalta en el mercado internacional por su capacidad pero que no necesariamente se apega a un lugar geográfico existen, tal es el caso del Basmati istaken cuya IGs está vinculada al continente africano (BLAKENEY, 2014, p.52).

\footnotetext{
3 En el caso del CUP en su actualización de 28 de septiembre de 1979, las denominaciones de origen aparecen brevemente enunciadas en el artículo $n^{\circ} 1$-definiendo que serán aplicables las disposiciones del artículo $n^{\circ} 9$ el que aborda cuestiones referentes a marcas y nombres comerciales. Por su parte, el artículo $n^{\circ} 10$, dispone que las protecciones establecidas en el artículo $n^{\circ} 9$ será aplicada en caso de utilización directa o indirecta de una indicación falsa concerniente a la procedencia del producto. Como se evidencia este artículo no aborda de manera expresa la definición de indicaciones de procedencia, ni denominaciones de origen

4 El Arreglo de Madrid de 1891 mantiene la represión de las indicaciones de procedencia falsa, escuetamente en el artículo 1.1 prohíbe importar productos que Ileven una indicación falsa o engañosa, resultandos indicados de manera sutil, sin que las denominaciones de origen aparezcan expresamente

5 El Arreglo de Lisboa de 1958 define en el artículo 2-. la denominación geográfica de un país, de una región o de una localidad que sirva para designar un producto originario del mismo cuya calidad o características se deben exclusiva o esencialmente al medio geográfico, comprendidos los factores naturales y los factores humanos

6 En el APDIC se define que las indicaciones geográficas son las que identifiquen un producto como originario del territorio de un miembro o de una región o localidad de ese territorio, cuando determinadas características del producto son imputables fundamentalmente a su origen geográfico.
} 
Entre indicaciones geográficas y patrimonio inmaterial en el caso havana club Janny Carrasco $\cdot$ Zadys Carrasco

Esta realidad, asevera que en la práctica las IGs, son un elemento relevante dentro del contexto de la globalización. La inmediatez de compras online, los flujos migratorios, la velocidad de transportación internacional han provocado que aquellos elementos locales que eran desconocidos puedan situarse a la par de sus competidores en el mercado internacional.

Gls are therefore simultaneously enabled by the consumer demand generated by global trade flows and potentially a means of resisting their homogenising effects. The international GI regime, contained in arts 22-24 of TRIPS, can be thematically summarised along the following lines (GANGJEE, 2012, p. 86).

Coincidiendo con Agdomar, es evidente el peligro de permitir la venta de productos falsificados o que indiquen un origen falso o engañoso, debido a que ello tiene un impacto directo en las identidades culturales y étnicas de las comunidades que tienen como principal sustento la producción y exportación de estos productos en el mercado internacional (AGDOMAR, 2007, p. 585).

Al respecto de esto, Gangjee refiere que las IGs, no tienen un efecto inmovilizador de los elementos culturales entorno a su elaboración y consumo, pues los productos están supeditados a la evolución propia del ámbito comercial y al igual que el resto de los derechos de propiedad intelectual, están sujetas a modificaciones, actualizaciones (GANGJEE, 2012, p. 85). En este sentido consideramos que es esencial su protección en lo relativo a la cuestión cultural, aun cuando ello acarre desafíos jurídicos difíciles de entender en el plano doméstico.

Por su parte (CHESMOND, 2007, p. 379) enfatiza que: “El aspecto cultural en ocasiones puede ser difícil de transmitir, ya que es a la vez sensible y personal, así como colectivo y simbólico, lo cual diferencia de los atributos usualmente claros y concisos relacionados al comercio y la economía que están presentes en el sistema internacional de protección a las IGs. 
Entre indicaciones geográficas y patrimonio inmaterial en el caso havana club Janny Carrasco $\cdot$ Zadys Carrasco

Aunque en una primera lectura puede pensarse que las IGs protegen exclusivamente productos o servicios, con el desarrollo del comercio internacional estos vienen acompañados de una tradición y origen del que no pueden escapar.

Más allá del aspecto jurídico las IGs traen aparejadas un nombre, un lugar, ciudad, región o país que las vinculad de manera indisoluble hacia la notoriedad internacional. Además, el producto que se coloca en el mercado es resultado del valor adquirido proveniente del patrimonio cultural inmaterial de un territorio.

De Acuerdo con la OMPI: por denominación de origen, se entiende un tipo especial de IG que se utiliza para productos que tienen cualidades específicas que se deben exclusivamente 0 esencialmente al entorno geográfico de la elaboración del producto. En el concepto de IG queda comprendido la denominación de origen (OMPI, 2014, p. 16-17)

Para entender más explícitamente esta realidad no remitimos a los estudios de varios autores como: (VIVEZ, 1932, p. 60), (MIRANDA, 2002, p. 272) donde son enunciadas las funciones que definen las IGs dentro del sistema internacional de la propiedad intelectual:

Tal como refiere (VIVEZ, 1932, p. 60) su primera función es identificativa: se diferencia un determinado producto o servicio de sus semejantes en el mercado, resaltando la región de procedencia como un elemento que define su calidad y la diferencia del resto de sus semejantes para los consumidores. En este sentido (MIRANDA, 2002, p. 272) resalta su factor diferenciador providenciando y valorando el origen geográfico como elemento diferenciador y determinante.

Yala función cualitativa no se debe expresamente a un elemento jurídico sino a proteger esa subjetividad que garantiza la conexión territorial con el mercado internacional, atribuyendo características que destacan su calidad, peculiaridad y representatividad de un territorio determinado. Un ejemplo que ilustra más claramente esta realidad es el caso del Tequila mexicano, el Havana Club (que 
Entre indicaciones geográficas y patrimonio inmaterial en el caso havana club Janny Carrasco • Zadys Carrasco

estudiaremos más adelante), el Vodka ruso por solo mencionar algunos ejemplos.

Y por último la función cultural, donde se reconoce el valor de las IGs por ser parte del fruto de un proceso tradicional, que emana de la producción local de u determinado territorio. Todo ello apoyado en la manera de hacer y producir desde el ámbito local, basado en experiencias y tradiciones que resalta el valor territorial y por ende cultural.

Esta protección local se vincula al aspecto del patrimonio cultural inmaterial que garantiza que procesos productivos sean transmitidos de generación en generación como mecanismo de perpetuidad de las tradiciones autóctonas. Este es determinado por un país, región o localidad donde que una vez que se hace notoriamente reconocido un producto o servicio, por pertenecer a un determinado lugar, no puede ser apartado en función de su vínculo con la calidad y representatividad del lugar.

Por otro lado, es característico en las IGs la confluencia de intereses públicosy privados. Entre los primeros resaltamos el valor colectivo que representan como: el desarrollo socioeconómico, el interés de los consumidores a los que esta figura da garantías y seguridad jurídica asociados a la calidad, minimización de la competencia desleal y el engaño; y los segundos enmarcados en las transacciones comerciales que devienen de la comercialización de estos productos fundamentalmente.

\section{Desentrañando los causes del Patrimonio Cultural Inmaterial}

El patrimonio es una construcción sociocultural que adquiere valor y sentido para aquel grupo que la realizó, heredó y aún conserva. Por ser una construcción sociocultural que se desarrolla en el tiempo el patrimonio es dinámico y cambiante. El patrimonio transmuta en el tiempo por transformaciones sociales y culturales 
Entre indicaciones geográficas y patrimonio inmaterial en el caso havana club Janny Carrasco $\cdot$ Zadys Carrasco

que actúan sobre él, y hacen que ese bien patrimonial pierda o adquiera nuevos valores o bien se apropie de nuevos significados y usos (LINA SARAI MEJÍA LÓPEZ, 2013, p. 56).

Según la Convención para la salvaguardia del patrimonio cultural inmaterial de 2003 en su artículo 2.1.- Se entiende por "patrimonio cultural inmaterial" los usos, representaciones, expresiones, conocimientos y técnicas -junto con los instrumentos, objetos, artefactos y espacios culturales que les son inherentesque las comunidades, los grupos y en algunos casos los individuos reconozcan como parte integrante de su patrimonio cultural. Este patrimonio cultural inmaterial, que se transmite de generación en generación, es recreado constantemente por las comunidades y grupos en función de su entorno, su interacción con la naturalezaysu historia, infundiéndoles un sentimiento de identidad y continuidad y contribuyendo así a promover el respeto de la diversidad cultural y la creatividad humana. A los efectos de la presente Convención, se tendrá en cuenta únicamente el patrimonio cultural inmaterial que sea compatible con los instrumentos internacionales de derechos humanos existentes y con los imperativos de respeto mutuo entre comunidades, grupos e individuos y de desarrollo sostenible (UNESCO, 2003, p. 2).

Por su parte (BENHAMOU Y THESMAR, 2011, p. 163) define que el patrimonio es: el entorno en el que vivimos, al que nos referimos o le atribuimos el simbolismo que le acompaña. En otras palabras, el patrimonio material tiene una dimensión inmaterial que es una fuente esencial de valor.

En las palabras de (ESPEITEX, 2004, p. 195) el patrimonio alimentario, apoyado en la definición de la UNESCO, es el conjunto de elementos materiales e inmateriales de las culturas alimentarias, establecido por la colectividad como una herencia del pasado. Este patrimonio integra concretamente el conjunto de productos agrícolas no procesados, procesados, los conocimientos y maneras de hacer en ese proceso productivos, así como todos los objetos, saberes y prácticas vinculados al consumo de un determinado producto y su distribución. 
Entre indicaciones geográficas y patrimonio inmaterial en el caso havana club Janny Carrasco $\cdot$ Zadys Carrasco

El patrimonio inmaterial ha ido ganando a lo largo del tiempo un uso identitario lo que establece una vinculación estrecha con el tema que nos ocupa las IGs y su relación con al patrimonio cultural inmaterial. Un ejemplo que ilustra con claridad esta relación es el caso del vino en Francia siendo que la Ley $n^{\circ} 1170$ - de 13 de octubre de 2014 dispone en su artículo n665-6.- el vino, producto de la vid, los territorios vinícolas, así como las sidras y las peradas, las bebidas espirituosas y las cervezas procedentes de tradiciones locales forman parte del patrimonio cultural, gastronómico y paisajístico protegido de Francia (FRANCIA, 2014, p. 13).

Es importante resaltar que esta práctica no es exclusiva de la legislación europea, sino que se ha extendido en el ámbito de las bebidas espirituosas como parte del patrimonio cultural inmaterial de los países que consideran la necesidad de protegerlas. Otros ejemplos encontramos en Brasil donde la Ley $n^{\circ} 8576$ de 24 de octubre de 2019 del Estado de Rio de Janeiro, declara patrimonio cultural histórico e inmaterial a la Caipirinha, bebida símbolo de Brasil (BRASIL, 2019, p. 1). Por su parte la UNESCO declaró la cerveza belga como parte de su patrimonio inmaterial de la humanidad entrando a la lista representativa del Patrimonio cultural inmaterial de la humanidad (UNESCO, 2016, p. 1).

Una de las principales críticas realizadas al tema del patrimonio inmaterial son las llamadas listas del Patrimonio Mundial de la Unesco donde autores como (MÁRQUEZ, 2012, p. 8) refieren que un mal planteamiento de los criterios puede llevar a un listado poco representativo.

Concretamente lo que sucede es que estas listas están sustentadas en nociones de monumentalidad, eurocentrismo y arquitectura de las elites del patrimonio mundial de los pueblos europeos y en especial de los grupos indígenas.

Por otra parte, debemos resaltar que los criterios de la UNESCO para determinar qué constituye patrimonio inmaterial se enmarcan dentro de un discurso multiculturalista que promueve la diversidad y la defensa de las expresiones de pueblos histórica 
Entre indicaciones geográficas y patrimonio inmaterial en el caso havana club Janny Carrasco • Zadys Carrasco

y políticamente excluidos cuyo objetivo es reforzar la identidad del Estado-Nación.

En ese sentido (MÁRQUEZ, 2012, p. 9) advierte que los Estadosnación promueven como patrimonio aquellas practicas que tienen un potencial especial para proyectar al país al extranjero (como la Celebración del Día de Muertos), por su aporte significativo a la economía local, lo que conlleva a que otras expresiones sean ignoradas por ser menos espectaculares o atrayentes.

Según reseña la Decisión 1. COM10 de la Unesco se elaboró una Nota de Orientación para la Confección de los Inventarios del Patrimonio Cultural Inmaterial. De Acuerdo con la Convención de Patrimonio Cultural en sus artículos 11.b y 12.1, donde se alude a la obligación de los Estados Partes a confeccionar inventarios del patrimonio cultural inmaterial.

Tal como refiere (HAFSTEIN, 2009, p. 14) la patrimonialización y su consagración en listas conlleva a una valoración y jerarquización de determinadas obras o expresiones a expensa de otras. Es decir, la patrimonialización establece una jerarquía de manifestaciones donde necesariamente unas son beneficiadas en detrimento de la exclusión de otras.

Es decir, el concepto de patrimonio cultural inmaterial y la manera en que este es implementado a escala global, demanda un análisis crítico que permita dar protección efectiva que pueda incluir otros elementos más de las prácticas de grupos indígenas o rurales que son definidas desde la óptica del mundo urbano y occidental como es el caso de las IGs. Además, el patrimonio cultural inmaterial en su interrelación con las IGs y el comercio internacional, necesitan de una interpretación interdisciplinar por parte de los países signatarios de esos instrumentos jurídicos y que hasta hoy no es muy frecuente. 


\section{Entendiendo las IGs y el patrimonio cultural inmaterial en la legislación cubana.}

\section{Desafío y oportunidades del ordenamiento jurídico interno}

Cuba desde la década del 1960 asume la posición de país socialista a escala global, lo que va a determinar de manera significativa su legislación interna ya sea en relación al derecho internacional como a la protección de la propiedad intelectual. Para teoría del sistema socialista del derecho internacional no aborda el problema de la relación entre el derecho interno e internacional desde la perspectiva del entendimiento doctrinario clásico que divide estas ramas del derecho y su relación en teoría monista y dualista.

Según los socialistas estas teorías no consiguen explicar de manera exhaustiva la relación existente entre esos ordenamientos jurídicos y se limitan a realizar una comparación entre ambos desde una postura formalista, dejando de un lado las leyes objetivas que gobiernan el desarrollo de ambas ramas del derecho, así como sus puntos comunes. Para los socialistas el derecho internacional y el derecho interno no tienen por qué contradecirse mutuamente, ni tener una relación de subordinación jerárquica (TUNKIN, 1979, p. 22).

Entre las principales limitaciones que observamos sobre la teoría socialista se destaca que existe una interpretación incompleta respecto a los avances de contenido formal entre monistas y dualistas y material en relación con la necesidad de armonización jurídica internacional e interna; negando así toda la práctica internacional. Otra de las limitantes es que inicia sus estudios desde la teoría abstracta sin materializarlo, en ningún ordenamiento jurídico concretamente, lo que dificulta la profundidad del análisis en relación a la integración de las normas internacionales en un contexto específico (USENKO, 1977, p. 87). 
Entre indicaciones geográficas y patrimonio inmaterial en el caso havana club Janny Carrasco $\cdot$ Zadys Carrasco

La primera Constitución del proceso revolucionario cubano (Constitución de 1975), tiene varias criticas respecto a la propiedad intelectual. Como ley suprema carecía de una alusión directa a la temática, ya que estos derechos no eran ni enunciados en el texto. Además, tampoco dejaba muy clara la manera en que eran implementados los instrumentos internacionales dentro del ordenamiento interno, así como tampoco a quien corresponde la interpretación de estos respecto a las normas internas.

De acuerdo con (PRIETO, 2018, p. 52) el control constitucional en la Carta Marga de 1975 al que estaban sometidos los tratados internacionales era político concentrado y posterior de las leyes en manos de la propia Asamblea Nacional del Poder Popular que aprueba estas mismas disposiciones, fundiéndose así controlador y controlado; lo que conllevaba a una ineficacia jurídica de las normas internacionales dentro del ordenamiento interno

Como fuentes reconocidas del Derecho Internacional, los tratados internacionales juegan un papel determinante en la definición de principios, derechos y obligaciones para los Estados que los suscriben. Partiendo de esta definición es importante delimitar que, en el ámbito cubano, los tratados internacionales en la Constitución de 2019 tienen un rango infra-constitucional según lo enunciado por el artículo 8, lo que sumado a la falta de un tribunal constitucional y la ausencia de la jurisprudencia como mecanismo de interpretación de la norma en caso de lagunas o ambigüedades; deja en total estado de vulnerabilidad tanto los instrumentos internacionales como su aplicación en el ámbito doméstico.

Esto contradice la postura de Cuba en el espacio jurídico internacionales sobre IGs ya que es signataria de varios instrumentos internacionales: Convenio de la Unión de Paris de 1883, Arreglo de Madrid de 4 de abril de 1891; Arreglo de Lisboa de 30 de octubre de 1958; Acuerdos de Propiedad Intelectual relacionados con el Comercio (ADPIC) de 1 de enero de 1995. Además de varios acuerdos bilaterales en el tema como son: Francia 1929, Alemania 1954, España 1979 y Perú 2000. 
Entre indicaciones geográficas y patrimonio inmaterial en el caso havana club Janny Carrasco $\cdot$ Zadys Carrasco

En el caso cubano, no existe de manera expresa en la legislación como son incorporados los instrumentos internacionales al ordenamiento jurídico nacional. Esta particularidad jurídica perjudica en ocasionesla adecuación de los tratadosinternacionales a las normas internas.

Un ejemplo que evidencia la realidad de lo anteriormente expuesto es el Decreto- Ley 208/2002 en su artículo 5 donde dispone que en el caso de las personas naturales o jurídicas extranjeras, serán aplicados los instrumentos internacionales con los que se tenga vínculo y la República de Cuba sea parte. Ante la ausencia de instrumentos internacionales, rige el principio de reciprocidad según las normas generales del Derecho Internacional. Solo que en este apartado la ausencia de un procedimiento específico para la implementación de los tratados internacionales en Cuba, deja al legislador en una postura de incertidumbre jurídica al carecer de elementos del derecho sustantivo que determinen hacia el orden interno la implementación del derecho internacional.

Sin embargo, un elemento positivo que apunta hacia las legislaciones más avanzadas en temas de IGs es el caso de la protección en el Decreto Ley n²08/2002 sobre patrimonio nacional. Esta postura resulta atrayente en la preservación de productos que constituyen parte de la identidad cubana como son el ron, el tabaco y el café, principales renglones exportables.

Entre las principales críticas al sistema jurídico cubanos resaltan la ausencia de un procedimiento específico sobre como registrar productos protegidos por el sistema IGs como elementos de la cultural inmaterial cubana y su preservación. La Ley $n^{\circledR} 1$ de 1977 de Patrimonio Cultural de la República de Cuba, así como su reglamento Decreto Ley 118 de 23 de septiembre de 1983; Resolución n9 de 2016 de la Presidenta del Consejo Nacional de Patrimonio Cultural de la República de Cuba; no dejan claro cuáles son los mecanismos jurídicos a desdoblar para otorgar la distinción a productos de esta naturaleza y como sería el procedimiento de proteger IGs en esta temática desde el ámbito del patrimonio cultural inmaterial. 
Entre indicaciones geográficas y patrimonio inmaterial en el caso havana club Janny Carrasco $\cdot$ Zadys Carrasco

Por otro lado, la legislación sobre patrimonio cultural inmaterial: "Instrucciones metodológica para el inventario automatizado del patrimonio inmaterial "Comidas y bebidas" del Consejo de Patrimonio Cultural Inmaterial, también presente algunas deficiencias relacionadas con las IGs que debemos resaltar.

Si bien está metodología hace referencia a aspectos como localización, zona, código de registro en el aspecto tipologías a nuestro criterio encontramos estos elementos no reciben un tratamiento adecuado de manera que constituyan una protección complementaria y significativa a la preservación de las IGs cubanas como el caso Havana Club. No basta el simple enunciado de la localización para determinar esta conexión jurídica, se hace necesario describir de manera más detallada aquellos elementos que otorgan particularidad y singularidad de un producto con su terruño como: características del suelo, tradiciones populares vinculadas a su preparación y significado en la cultura local.

Tal como mostramos anteriormente, esta jerarquización de la patrimonialización definida por listas trae consigo un divorcio persistente entre patrimonio cultural inmaterial y las IGs, pues aquellos elementos que podrían ser susceptibles de ambas protecciones; no ganan las debidas garantías en la legislación de patrimonio inmaterial cubana.

Otroelementoadestacarenelcasodecubanosonlasinstituciones reconocidas como patrimonio mundial de la humanidad, en su gran mayorías espacios históricos, arquitectónicos, naturales, tradiciones musicales, entre otros ${ }^{7}$. El modelo cubano evidencia esa persistencia en enmarcar con mayor connotación jurídica a sitios históricos o turísticos, dentro del ámbito de protección y en menor medidas a productos gastronómicos.

\footnotetext{
7 Las instituciones cubanas reconocidas como patrimonio mundial de la humanidad son : Parrandas de la región central de Cuba, El punto, La rumba cubana, mezcla festiva de baile y música, y todas las prácticas culturales inherentes, Centro Histórico Urbano de Camagüey, Centro Histórico Urbano de Cienfuegos, Tumba Francesa

Parque Nacional Alejandro de Humboldt, Paisaje Arqueológico de las Primeras Plantaciones Cafetaleras en el Sudeste de Cuba, Parque Nacional Desembarco del Granma, Valle de Viñales, Castillo San Pedro de la Roca de Santiago de Cuba, Centro Histórico de Trinidad y su Valle de los Ingenios, Centro Histórico de La Habana Vieja y su Sistema de Fortificaciones Coloniales. Disponible en : http://www.cnpc.cult.cu/patrimonios-mundiales. Consultado el 7 de abril de 2021.
} 
Entre indicaciones geográficas y patrimonio inmaterial en el caso havana club Janny Carrasco • Zadys Carrasco

Si bien la Resolución n9 de 2016 de la Presidenta del Consejo Nacional de Patrimonio Cultural de la República de Cuba ${ }^{8}$, reconoce como parte del patrimonio cultural inmaterial la actividad de los maestros roneros, esto no resulta suficiente para concatenar la práctica jurídica de las IGs y el patrimonio cultural inmaterial en la legislación cubana.

Entre las principales críticas que resalta en este divorcio jurídico, resulta evidente que en ninguna de las etiquetas utilizadas en la bebida Havana Club, se hace alusión a la actividad de los maestros roneros, como parte del patrimonio inmaterial cubano. Esto provoca que técnicamente este producto cubano no consiga resguardar de manera incuestionable, ese arraigo que envuelve el ron cubano, como un producto de verdadera identidad nacional, tradición y cultura tradicional. Esta realidad evidencia que en el ámbito internacional el Havana Club haya enfrentado varios procesos judiciales que podrían haber sido evitados de tener esa distinción en su IGs, tal como abordaremos más adelante.

Al mismo tiempo en la lista nacional de patrimonio inmaterial en la categoría de Comidas y Bebidas ${ }^{9}$ tampoco se reconoce el ron como una bebida espirituosa que representa un conjunto de prácticas y tradiciones genuinamente cubanas, lo que contradice con la Resolución n9 de 2016 supra citada al reconocer la actividad ronera como parte del patrimonio inmaterial cubano

Tal como observamos en la legislación de patrimonio cultural inmaterial las IGs no son entendidas con un efecto movilizador de elementos culturales que involucre su elaboración y consumo. Su protección está supeditada a la evolución propia del contexto comercial y al igual que el resto de los derechos de propiedad intelectual es susceptible de modificaciones, abandono y desprotección. El aspecto cultural puede en ocasiones ser difícil de entender desde el contexto de las IGs en la realidad cubana, debido a que envuelve una mezcla de conocimiento tradicional y

8 En la tradición de fabricación del ron cubano ligero tienen un papel preponderante los maestros roneros, quienes son portadores de conocimientos y técnicas donde se combinan ciencia, tradición y sensibilidad, y que convierten a cada ron de la Denominación de Origen Cuba en un exquisito producto, reconocido como único en el mundo

9 Para mayor información consultar la lista del Centro de Patrimonio Cultural de la República de Cuba. Disponible en: http:// www.cnpc.cult.cu/comidas-y-bebidas. Consultado el 12 de abril de 2021. 
Entre indicaciones geográficas y patrimonio inmaterial en el caso havana club Janny Carrasco $•$ Zadys Carrasco

simbolismo cultural colectivo que no alcanza una protección eficaz en el ordenamiento interno.

\section{Tierra, autenticidad y sabor: "el terroir" / "terruño"}

Tal como describe (MARCHENAY, 2006, p. 110) el terroir es una palabra de origen francés que, en su traducción semántica al español, acaba perdiendo su verdadera esencia jurídica. El terroir está relacionada a propiedades biofísicas únicas de ciertos lugares especies y plantas nativas y tipos de suelos.

De acuerdo (MARCHENAY, 2006, p. 115) gran parte de la originalidad de un producto, es resultado de su vínculo directo con el terroir, considerado por los estudiosos del tema como una especie de coautor, le terroir es coautor de la producción por imprimir aquellas características únicas que el suelo, las plantas y la tradicional de cultivo imprimen como distinción de calidad. Esta realidad vincula las IGs como elemento indispensable en la relación con la cultura inmaterial. El terror o terruño vincula aspectos sociales e históricos que determinan la singularidad, irrepartibilidad y autenticidades presentes en la producción y comercialización de cualquier patrimonio gastronómico que trasciende las fronteras físicas de los Estados y se posiciona en el mercado global internacional.

Entre los principales desafíos que encuentra el terruño en el caso Havana Club encontramos que: no existe una política estatal que evoque al terruño como vínculo indispensable que conecta patrimonio inmaterial y las IGs. Las principales distinciones hacen referencia a cuestiones de calidad, método de destilación o grados de alcohol, obviando de manera fehaciente la importancia de esta figura dentro de la autenticidad de un producto que se distingue en el mercado internacional por su origen cubano.

En este sentido no es pertinente entender la protección jurídica de las IGs y el patrimonio inmaterial desvinculados del terror o 
Entre indicaciones geográficas y patrimonio inmaterial en el caso havana club Janny Carrasco • Zadys Carrasco

terruño. Hoy la legislación cubana como hemos venido resaltando es omisa en establecer este vínculo imprescindible con el terruño como elemento indispensable para la protección de las IGs y su relación con el patrimonio inmaterial.

Sin embargo, en la interrelación IGs y patrimonio cultural inmaterial esto no resulta suficiente, resaltando las principales críticas a la etiqueta ${ }^{10}$ como elemento distintivo e informativo de referencia para el consumidor:

- No se hace referencia a la tradición ronera reconocida como parte del patrimonio cultural inmaterial cubano.

- No refiere las condiciones del suelo donde son cultivados las materias primas para la fabricación del producto. Como es el caso de la caña de azúcar, principal materia prima de este producto.

- No resalta el valor cultural de esta bebida espirituosa dentro de la cultura popular cubana.

De manera, significativa estas ausencias condicionan el incremento de falsificación del producto en el mercado internacional. Además, esto refuerza la necesidad de entender la relación de las IGs con el patrimonio inmaterial como un elemento imprescindible para su protección en el ámbito del comercio internacional y el ordenamiento jurídico cubano.

\section{Tras los causes judiciales del Havana Club como IGs y Patrimonio Inmaterial}

Apenas 90 millas, aproximadamente $180 \mathrm{~km}$, dividen dos sistemas jurídicos extremos. Desde la llegada de la Revolución

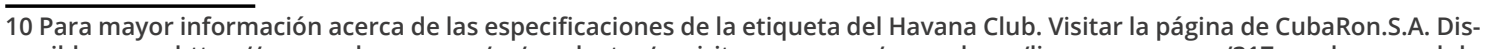
ponible en: https://www.cubaron.com/es/productos/espirituosos-menu/ron-cubano/lineas-ron-menu/317-ron-havana-club. Consultado el 21 de abril de 2021. 
Entre indicaciones geográficas y patrimonio inmaterial en el caso havana club Janny Carrasco $\cdot$ Zadys Carrasco

Cubana, los E.U.A y Cuba han enfrentado una guerra económica que trasciende en el tiempo. En cuestiones de propiedad intelectual esta realidad ha sido un poco diferente, más de 5000 marcas norteamericanas han sido registradas en Cuba y más de 400 cubanas registradas en E.U.A.

El caso Havana Club ha representado litigios durante más de una década y numerosas determinaciones judiciales y administrativas ${ }^{11}$, relacionadas al derecho sobre la marca. Particularmente en este caso destacan las reiteradas violaciones a las IGs de las bebidas espirituosas pactadas en los Acuerdos de Propiedad Intelectual Dedicados al Comercio (ADPIC) y del que ambos países son signatarios.

En este sentido analizaremos la decisión Pernod Ricard USA, LLC v. Bacardí U.S.A., Inc., 653 F.3d 241 (2011), por su estrecha relación con la IGs y el patrimonio cultural inmaterial a fin de demostrar jurídicamente la vinculación de estos elementos y su impacto en los litigios internacionales como este caso.

La historia Havana Club se remonta al siglo XIX en Cuba donde la familia Arrechabala producía ron bajo la marca Havana Club, vendiéndolo localmente y exportándolo a E.U.A. En 1960 con el proceso de nacionalización de la Revolución Cubana, se expropia el negocio. Tres años después comienza a ser aplicado el Embargo económico de E.U.A para los productos cubanos; pero a pesar, de ello en 1976 Cubaexport (Empresa encargada de las Exportaciones Cubanas en los E.U.A) logra registrar la marca Havana Club en la Oficina de Patentes y Marcas en E.U. A ${ }^{12}$. En 1994, el gobierno cubano transfiere la Marca a Pernod Ricard, siendo revocada la transferencia por la Oficina de Control de Activos Extranjeros (OFAC) de los Estados Unidos por lo que permanece con Cubaexport hasta el 2006, cuando es denegado el permiso para renovar la marca a este titular.

\footnotetext{
11 A modo de explicación para situar al lector colocamos una breve reseña jurídica de las principales decisiones judiciales. Havana Club Holding, S.A. v. Galleon S.A., 203 F.3d 116 (2d Cir.2000); Havana Club Holding, S.A. v. Galleon, S.A., 62 F.Supp.2d 1085 (S.D.N.Y.1999); Havana Club Holding, S.A. v. Galleon, S.A., 1998 WL 150983 (S.D.N.Y. Mar. 31, 1998); Havana Club Holding, S.A. v. Galleon, S.A., 961 F.Supp. 498 (S.D.N.Y.1997); Havana Club Holding, S.A. v. Galleon, S.A., 974 F.Supp. 302 (S.D.N.Y.1997).

12 Este registro resulta necesario por el Sistema Internacional de Marcas para la comercialización de un determinado producto en un territorio determinado.
} 
Entre indicaciones geográficas y patrimonio inmaterial en el caso havana club Janny Carrasco • Zadys Carrasco

Entre las principales discusiones del caso en 2011 resaltamos que las partes, alegando publicidad engañosa, buscan permanecer con el derecho exclusivo de la palabra que identifica el producto "Havana Club", a lo que Pernod Ricard alega ser un caso de competencia desleal debido a que el nombre es notoriamente reconocido por ser de origen cubano.

A los efectos de esto es planteado por la corte una interrogante crucial: ¿Es el origen geográfico más parecido al patrimonio o a la fuente de producción? A lo que la Corte Suprema entendió que el caso se refiere concretamente al origen del producto y su vinculación con las ventas y no al autor de ninguna idea, aplicando el enfoque de interpretación de origen geográfico, alegando la importancia del lugar de fabricación en lugar de la fuente de la receta fruto de esa herencia a la que ha hecho referencia Bacardí durante varios años en los diferentes procesos judiciales. Tal como destaca el tribunal en su decisión:

And viewed in that context, any thought a consumer might
have that the words "Havana Club" indicate the geographic
origin of the rum must certainly be dispelled by the plain and
explicit statements of geographic origin on the label (CIRCUIT,
2011, p. 5).

Esta interpretación de la Corte evidencia la necesidad de entender las IGs desde el ámbito del patrimonio cultural inmaterial, sobre todo aquellos productos que han alcanzado una notoriedad internacional debido a su procedencia como es el caso de Havana Club y del cual no se pueden desprender.

El Havana Club es un producto cubano que se remonta a cuestiones de tiempo, tradición, suelo, condiciones climáticas, pericia de la clase campesina que les permite cultivar y obtener estándares de calidad que sobrepasan la relación familiar que dio origen a la marca. Su valor agregado en el respeto al medio ambiente y el valor de la tierra son características que aun cuando 
Entre indicaciones geográficas y patrimonio inmaterial en el caso havana club Janny Carrasco $\cdot$ Zadys Carrasco

no ganan destaque en la etiqueta del producto están en el arraigo de la cultura inmaterial cubana.

Es valido resaltar que los productos protegidos por IGs alcanza en el mercado internacional un precio superior a un 20 o 30\% de sus semejantes que no poseen un signo distintivo vinculado a tradiciones y territorios. En este sentido debemos entender que los principales litigios que ha enfrentado esta IGs se deben al valor comercial y no a la conexión con las tradiciones, historias y maneras de hacer ron. De ahí que la protección jurídica de las Estados a estos productos sea elevada. Sin embargo, en el caso cubano hemos ido resaltando que la ausencia de un entendimiento interdisciplinar sobre el tema ha acarreado infortunios jurídicos.

Es importante resaltar que el caso Havana Club posee una fuerte carga política vinculada al embargo económico aplicado por los E.U.A a los productos cubanos, que persiste como una política injerencista y arbitraria en relación los principios generales del derecho internacional, tales como la no intromisión de los asuntos internos y el respeto a la identidad y el patrimonio cultural inmaterial. Lo que deviene en un incumplimiento indirecto de las obligaciones internacionales sobreponiendo intereses políticos a compromisos jurídicos.

Otra importante crítica a las decisiones judiciales del caso Havana Club recaen sobre el entendimiento de que la legislación norteamericana no posee de manera clara que las IGs sean vinculadas en determinados casos concretos a cuestiones del patrimonio cultural inmaterial, por su vinculación a las tradiciones y arraigo al terruño. Está postura evidencia la necesidad de comenzar a entender las IGs desde su enfoque cultural, debido a que la representatividad de una marca con arraigo a un territorio es más que una cuestión puramente comercial, su ámbito se desplaza hacia la identidad local que no puede ser desvinculada por cuestiones puramente comerciales.

En resumen, el caso "Havana Club" muestra dos grandes problemas en el nivel de protección ofrecida por la norma 
Entre indicaciones geográficas y patrimonio inmaterial en el caso havana club Janny Carrasco • Zadys Carrasco

internacional Acuerdos de Propiedad Intelectual relacionados al Comercio (ADPIC) acerca de las IGs. Primero la ausencia de una conexión directa con la Convención de Patrimonio Inmaterial de la Unesco de 2003, en la referente a garantizar para las IGs esta dualidad de protección. Segundo la fragilidad en entender las IGs como elemento estrechamente vinculado al terruño y de imposible desvinculación sea por cuestiones comerciales, sea por litigios transnacionales.

\section{Conclusiones}

Las IGs en el contexto internacional permite constatar que su protección jurídica es relativamente reciente, lo que viene a impactar de manera directa e infeliz en el ordenamiento jurídico interno, como es el caso cubano. Si bien las IGs hace referencia a identificación de productos en su conexión con lugares geográficos, se observa que no existe un consenso en que este concepto deba de manera inequívoca conferir un derecho.

Aunque las IGs deben referir protección vinculada al lugar de origen, lo cierto es que para los productores y exportadores estas no son más que factores de promoción que pocas veces son aprovechados de manera eficiente, tal como demuestra el caso Havana Club.

La justificación cultural refiere sin dudas que las IGs aluden al efecto de protección y preservación de tradiciones, costumbres y maneras de hacer. Sin embargo, la ausencia de una conexión entre estos dos elementos provoca que estas solo sean protegidas desde el ámbito comercial, denigrando su contribución a las expresiones culturales tradiciones e inmateriales.

La existencia de diversas jurisdicciones sobre la protección de las IGs, como el caso de los E.U.A y Cuba, evidencia la necesidad de evaluar las respuestas jurídicas dadas en relación con sus aspectos vinculados al patrimonio cultural inmaterial, siendo necesario una 
Entre indicaciones geográficas y patrimonio inmaterial en el caso havana club Janny Carrasco $•$ Zadys Carrasco

protección multidisciplinar que garantice un verdadero amparo a las IGs.

El caso Havana Club es sin dudas, un reflejo de la necesidad jurídica de entender las IGs desde el patrimonio cultural inmaterial. Debido a los valores del terruño, tradiciones roneras y apego a la cultural local, la protección de esta IGs representa la conexión global y local del producto cubano más reconocido internacionalmente.

\section{Bibliografía}

Accioly, Hildebrando. Direito Internacional Público. 20. São Paulo: Saraiva, 2018.

AGDOMAR, Michelle. Removing the Greek from Feta and Adding Korbel to Champange: the Paradox of Geographical Indications in International Law. Fordham Intellectual Property Media and Enterntainment Law Journal, 2007, p. 541-608.

Ana Carvalho, Christian Hotti. Le patrimoine culturel immatériel: Premiéres expériences en France. ETNOGRÁFICA, 2013, p. 430-432.

Arizpe, Lourdes. Los Debates internacionales en torno al patrimonio cultural inmaterial. Centro Regional de Investigaciones

Multidisciplinarias - UNAM 13, no. 38 (septiembre-diciembre 2006), p. 16.

Barbero, Jesús Martín. La globalización en clave cultural: una mirada latinoamericana. Coloquio Internacional Globalismo y Pluralismo. Montreal, 2002. 26.

Bassos, Maristela. O Direito Internacional da Propriedae Intelectual. 1era. Vol. 1. Porto Alegre: Livraria do Advogado, 2000. Benhamou, Françoise, and David Thesmar. Valoriser le patrimoine culturel de la France. Unesco, Paris, 2011, 162. 
Entre indicaciones geográficas y patrimonio inmaterial en el caso havana club Janny Carrasco $•$ Zadys Carrasco

Blakeney, Michael. Geographical Indications: What Do They Indicate? The WIPO Journal: Analysis of Intellectual Property Issues. 2014, p. 50-56.

Brasil. Lei 8576/2019 de 23 de outubro de 2019. Rio de Janeiro: Diário Oficial, 2019, p. 2.

CHESMOND, Rhonda. Protection or privatisation of culture? The cultural dimension of the international Intellectual Property debate on geographical indications of origin. European Intellectual Property Review, no. 9, 2007, p. 379-388.

Circuit, United States Court of Appeals for the Third. Pernod Ricard USA, LLCv. Bacardi U.S.A, Inc., 653 F.3d241(2011). United States Court of Appeals for the Third Circuit, 4 de Agosto de 2011, 22.

E.U.A. Lanham Act. n.d.

Espeitex, Elena. Patrimonio alimentario y turismo: una relación singular. Pasos 2, 2004, p. 193-213.

EUA, Departamento de Estado. $§ 515.582$ Importation of cetain goods and services produced by independent Cuban entrepreneurs. https://www.state.gov/the-state-departments-section515-582-list/. Washintong DC, 2015.

Francia. Loi n²014-1170. Journal Officiel de la République Française. Paris: legifrance.gouv.fr, 2014. 79.

Gangjee, Dev S. Geographical Indications and Cultural heritage. The WIPO Journal, abril 2012, p. 11.

Hafstein, Valdimar. Intangible heritage as a list: from masterpieces to representation. Edited by Natsuko Akagawa Laranje Smith. Vol. 1er. New York: Routledge, 2009.

James, Erik. Private International Law. Haya: HCCH, 2010.

Lina Sarai Mejía López, Miriam Bravo Rodríguez, Sonia Edith Mejía Castillo. La gastronomía como símbolo en la cultura. Culinaria. Revista virtual especializada en Gastronomía 2013, p. 50-64. 
Entre indicaciones geográficas y patrimonio inmaterial en el caso havana club Janny Carrasco $•$ Zadys Carrasco

Marchenay, Laurence Bérard and Philippe. Local products and geographical indications:taking account of local knowledge and biodiversity. International Social Science Journal. Cultural Diversity and Biodiversity (2006), p. 09-116.

Márquez, Isabel Villaseñor Alonso y emiliano Zolla. Del patrimonio cultural inmaterial o la patrimonialización de la cultura. Cultura y representaciones sociales, 2012.

Miranda, Pontes De. Tratado de Direito Privado. 1era. Campinas: Bookseller, 2002.

OMPI. Las Indicaciones Geográficas. Ginebra : OMPI, 2017.

OMPI. Principios básicos de la Propriedaad Industrial. OMPI No895(S). Ginebra: OMPI, 2014. p. 16-17.

Prieto, Marta Valdez. Del Control constitucional en Cuba. Propuestas necesarias. Cuestiones Constitucionales. Revista Mexicana de Derecho Constitucional, 2018, p. 43-64.

Riley, Michael. Cigars and Rum: Hazardoust to the Health of Intellectual Property Law? How the Cohiba Cigar and Havana Club Rum Cases Reveal a Carve-Out for the Intellectual Property Disputes with a Cuba Nexus. University Of Miami InterAmerican Law Review, 2007, p. 44.

Santiago, Yanis Rosana Blanco. LA Protección de las Indicaciones Geográficas en el Comercio Internacional: Recepción y Efectividad en el Ordenamento Jurídico Puertorriqueño. Vol. 1. Madrid: Universidad Complutense Madrid, 2017.

Tunkin, G. I. Curso de Derecho Internacional. Moscú : Progreso, 1979.

UNESCO. 11. COM. Cerveza Belga en la Lista del Patrimonio Cultural Inmaterial de la Humanidad. Paris: UNESCO , 2016. 2.

UNESCO. Convención para la Slavaguardia del Patrimonio Cultural Inmaterial. Paris: UNESCO, 2003. 
Entre indicaciones geográficas y patrimonio inmaterial en el caso havana club Janny Carrasco $•$ Zadys Carrasco

Unit States Court of Appeals, Second Circuit. Havana Club Holding S.A v. Galleon S.A. Febrero 2000.

Usenko, E. T. Problemas teóricos en la relación del derecho internacional y el derecho interno. Anuario Soviético de derecho internacional, 1977, p. 87 y sigs.

Vicente, Darío Moura. A tutela internacional da propriedade intelectual. Coimbra: Almedina, 2008.

Vivez, Jacques. Les appellations dórigine-Législation et Jurisprudence Actuelles. 1era. Bordeaux: Faculté de Droit de L'Université de Bordeaux , 1932.

WOLF, David B. Effective Protection against Unfair Competition under Section 44 of the Lahham Act, Trademark Rep. 1992, p. 33-57. 